\title{
Mechanical strain modulates age-related changes in the proliferation and differentiation of mouse adipose-derived stromal cells
}

\author{
See-Chang Huang ${ }^{1}$, Tzu-Chin Wu², Hsiao-Chi Yu², Mei-Ru Chen², Chun-Min Liu', Wen-Sheng Chiang',
} Kurt M Lin'2,3*

\begin{abstract}
Background: Previous studies on the effects of aging in human and mouse mesenchymal stem cells suggest that a decline in the number and differentiation potential of stem cells may contribute to aging and aging-related diseases. In this report, we used stromal cells isolated from adipose tissue (ADSCs) of young (8-10 weeks), adult (5 months), and old (21 months) mice to test the hypothesis that mechanical loading modifies aging-related changes in the self-renewal and osteogenic and adipogenic differentiation potential of these cells.

Results: We show that aging significantly reduced the proliferation and increased the adipogenesis of ADSCs, while the osteogenic potential is not significantly reduced by aging. Mechanical loading (10\% cyclic stretching, 0.5 $\mathrm{Hz}, 48 \mathrm{~h}$ ) increased the subsequent proliferation of ADSCs from mice of all ages. Although the number of osteogenic colonies with calcium deposition was increased in ADSCs subjected to pre-strain, it resulted from an increase in colony number rather than from an increase in osteogenic potential after strain. Pre-strain significantly reduced the number of oil droplets and the expression of adipogenic marker genes in adult and old ADSCs. Simultaneously subjecting ADSCS to mechanical loading and adipogenic induction resulted in a stronger inhibition of adipogenesis than that caused by pre-strain. The reduction of adipogenesis by mechanical strain was loadingmagnitude dependent: loading with $2 \%$ strain only resulted in a partial inhibition, and loading with $0.5 \%$ strain could not inhibit adipogenesis in ADSCs.

Conclusions: We demonstrate that mechanical stretching counteracts the loss of self-renewal in aging ADSCs by enhancing their proliferation and, at the same time, reduces the heightened adipogenesis of old cells. These findings are important for the further study of stem cell control and treatment for a variety of aging related diseases.
\end{abstract}

\section{Background}

Recent findings on age-related changes in adult stem cells and stem cell niches suggest that the aging process and aging-related diseases may involve age-dependent stem cell loss, including alterations in their numbers and/or differentiation potential, although many of the details are still not understood [1]. One example of aging-related diseases is osteoporosis in the elderly, in which bone loss and increased bone marrow fat may result from reduced osteogenic potential and a tilted

\footnotetext{
* Correspondence: klin@nhri.org.tw

${ }^{2}$ Division of Medical Engineering Research, National Health Research
} Institutes, Zhunan Town, Miaoli, Taiwan osteogenic/adipogenic balance in bone marrow mesenchymal stem cells (BMMSCs) [2,3]. Another example is obesity, which can be a risk factor for diabetes and cardiovascular diseases and involves excess accumulation of white adipose tissue that is differentiated from MSCs [4]. Most of the current knowledge regarding adult stem cells derives from studies of MSCs isolated from bone marrow. Displaying similar differentiating potentials to BMMSCs, ADSCs possess clear advantage in clinical uses due to easy and repeatable access as well as simple isolation and expansion procedures that promise broad applications for cell therapy and tissue engineering $[5,6]$. Age-related declines in the lifespan, proliferation, and differentiation capacity of 
human and mouse BMMSCs have been reported previously [7-12], but most of the mechanisms are still unclear. Aging effects in human and murine ADSCs were only partially explored, and it appears that the osteogenic differentiation capacity of ADSCs is maintained with aging $[5,13]$.

Effects of mechanical force on growth rate, signal transduction, and cell phenotype have been widely documented in a variety of cell types. Mechanical loading was reported to induce osteogenic differentiation [14-17] and smooth muscle cell differentiation [18-20] in BMMSCs, or lead to inhibition of adipogenesis [14] through durable $\beta$-catenin activation [21]. In growing mice, exposure to low-magnitude mechanical signals alters the cell fate of BMMSCs by inhibiting adipogenesis [22]. Uniaxial strain inhibited the proliferation of human ADSCs and the expression of early smooth muscle cell markers [23]. In this study, we looked for agingrelated differences in ADSCs isolated from young, adult, and old mice. By applying mechanical strain, we tested the hypothesis that mechanical loading counteracts the effects of aging by modulating the self-renewal and differentiation potential of murine ADSCs.

\section{Results}

\section{Age-related changes in mouse ADSCs}

To explore the effect of donor age on ADSCs, we isolated ADSCs from a stromal-vascular cell fraction (SVF) derived from the gonadal fat pads of young (8-10 weeks), adult (5 months), and old ( 21 months) mice. The proliferation rate of passage zero (P0) ADSCs was studied by measuring their average doubling time. The doubling time of ADSCs isolated from young mice was significantly shorter than that of ADSCs from old mice (Figure 1A). Proliferation of ADSCs was also measured by a colony forming assay in which we plated 5000 young, adult, or old P0 ADSCs and counted the colonies formed and the number of cells in each colony after 5 or 8 days of culture. The large colonies (> 50 cells per colony) are of particular importance because they are formed by the most active ADSCs with sustained proliferating ability. This assay demonstrated that young ADSCs formed significantly more large colonies than either adult or old ADSCs (Figure 1B).

To test the differentiation potential of ADSCs, P1 cells were subjected to osteo-, myo-, and adipogenic induction for 21 days followed by von Kossa, Liu's, or OilRed O staining (Figure 1C). Compared to young ADSCs, old ADSCs exhibited reduced von Kossa staining and formed fewer multinucleated myotubes, indicating a reduction in calcium deposition and myogenic differentiation. The Oil-Red O staining of old ADSCs was significantly stronger than young ADSCs, indicating that the adipogenic potential was elevated by aging.
We measured the expression of bone marker genes, Runx2 and Bglap1, in young and old ADSCs following 21 days of osteogenic induction. Induction with osteogenic medium led to an increase in the expression of Runx2 and Bglap1 in both young and old ADSCs compared to non-induced cells. However, the expression of these bone marker genes, after being normalized to the GAPDH expression, was not significantly different between young and old ADSCs (Figure 2A). This result suggests that fewer von Kossa-stained colonies were formed by old ADSCs likely due to a reduction in the number of colonies, but not a reduction in the osteogenic potential of old ADSCs. In contrast, following adipogenic induction there was an age-dependent increase in the expression of the AP2 and PPAR $\gamma$ genes (Figure 2B), indicating a higher capacity for adipogenesis in old ADSCs.

\section{Mechanical loading counteracts aging-dependent changes \\ Pre-exposure to mechanical strain increases ADSC self- renewal}

To investigate the effect of mechanical loading, we subjected P2 ADSCs to $48 \mathrm{~h}$ of equibiaxial cyclic strain (in a $0.5 \mathrm{~Hz}$ sinusoidal waveform with a maximum strain of $10 \%$ ) and, after a subsequent passage, assessed their colony-forming ability. As shown in Figure 3A and 3B, 48 $h$ of mechanical loading led to a significant increase in the number of colonies formed by ADSCs. This increase in colony number was more pronounced in adult and old ADSCs than in young ADSCs, likely due to the high basal colony-forming ability of unstimulated young ADSCs.

Pre-exposure to mechanical strain increases calcium deposition, but not osteogenic potential in ADSC colonies

To investigate osteogenic differentiation in ADSCs preexposed to mechanical strain, the ADSCs were replated following mechanical loading and induced by osteogenic induction medium for various periods of time. As shown in Figure 4A, ADSCs from young, adult, and old animals exhibited an age-related decrease in AlizarinRed-S (ARS) staining, confirming the result shown in Figure 1C. Pretreatment with mechanical strain appears to result in an increase in ARS staining. However, the detailed analysis shown in Figure 4B demonstrated similar ARS staining density in samples with or without prestrain. Aging remains the only determinant factor of ARS staining intensity. We also measured the expression of osteogenic marker genes in ADSCs after 21 days of osteogenic induction and found that Runx2 or Bglap1 expression was not significantly altered by pre-strain, except that Runx2 expression was enhanced slightly in pre-strained young ADSCs. These data suggest that prestrain did not significantly induce osteogenesis of ADSC 


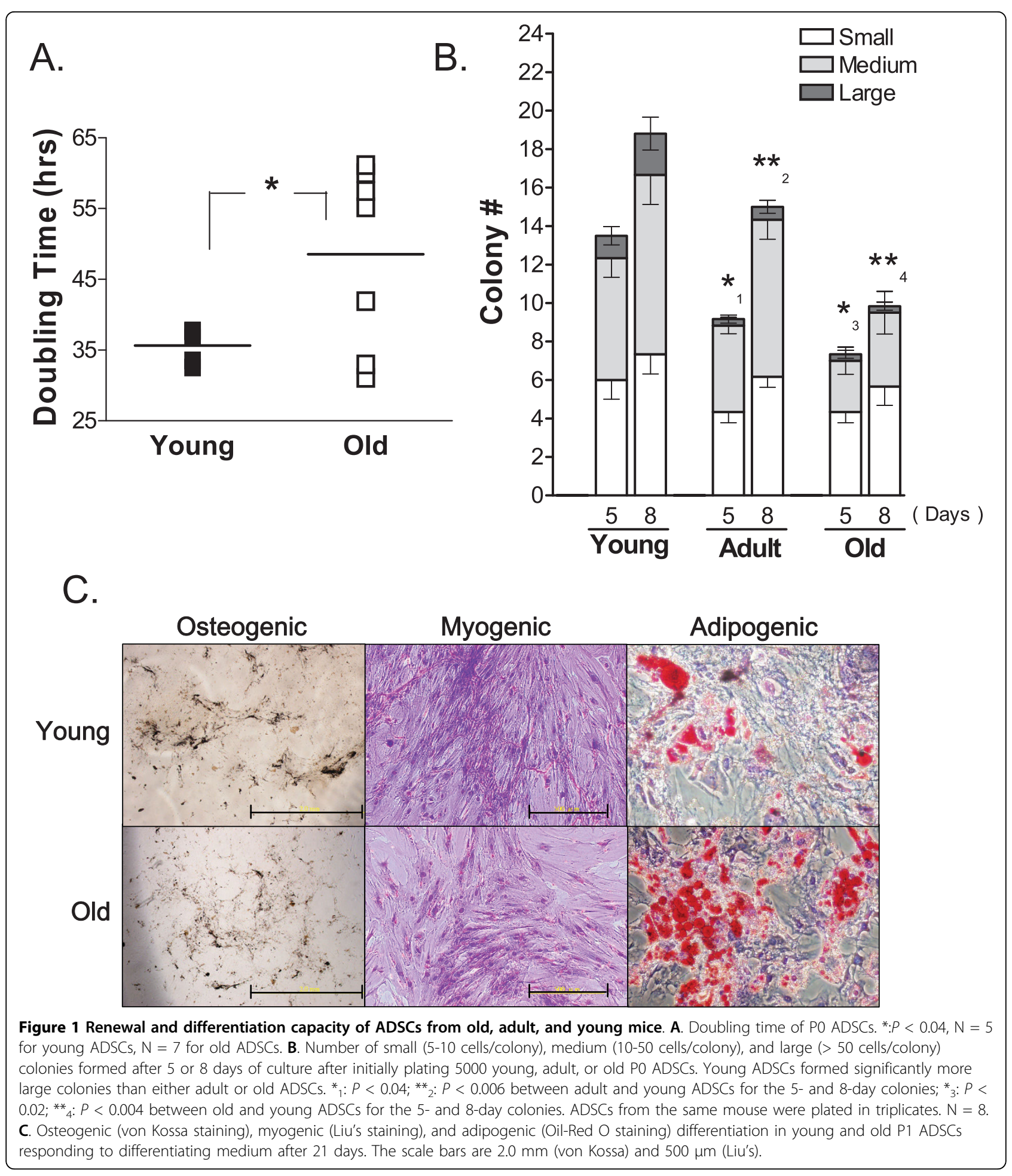




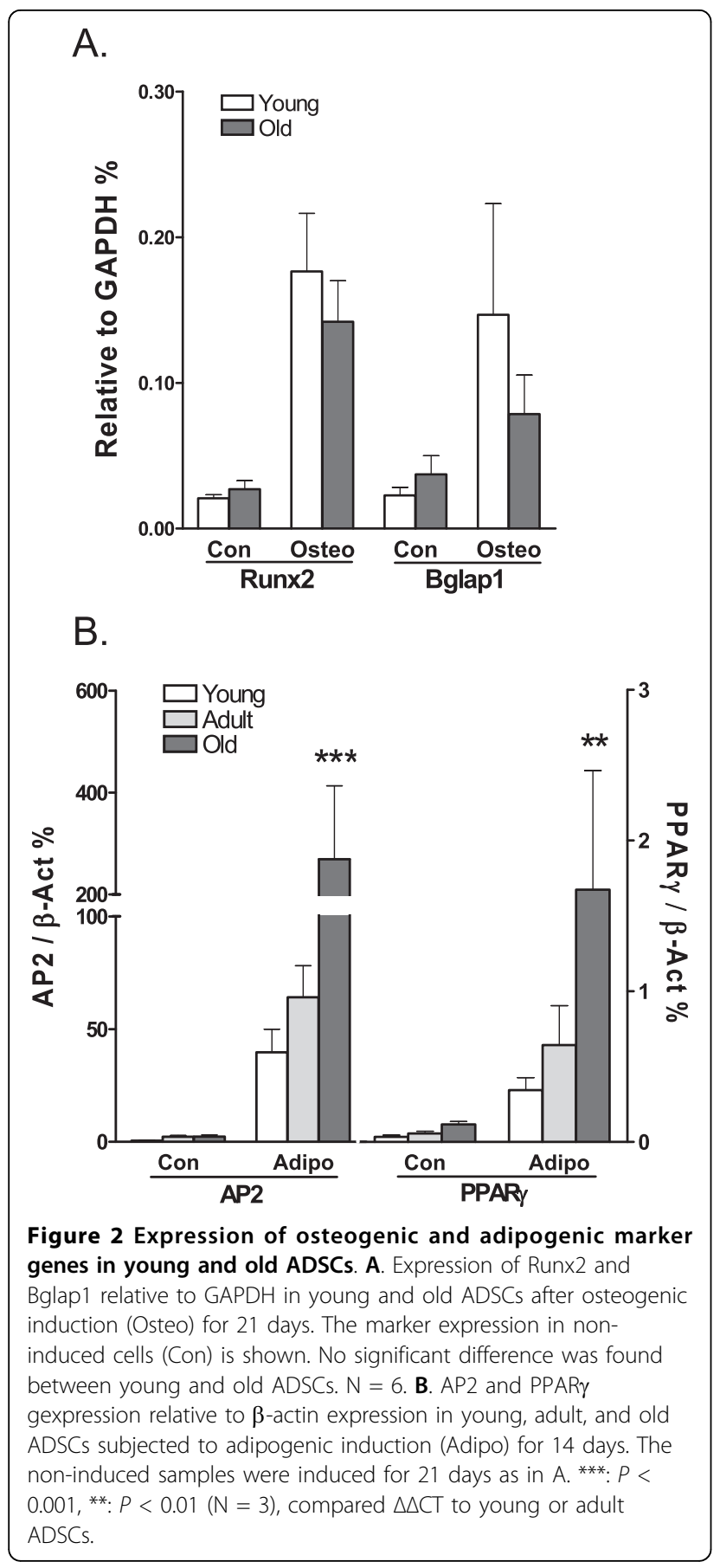

colonies. We further confirmed this conclusion using quantitative ARS staining after osteogenic induction in adult ADSCs. We quantified the staining results by measuring the absorbance of extracted ARS staining and the fluorescence emitted by ethidium bromide, which stains the DNA and is used as an indirect indication of the cell number contained in each culture well. As shown in Figure 5A, the normalized ARS absorbance gradually increased in osteogenically induced ADSCs, but did not

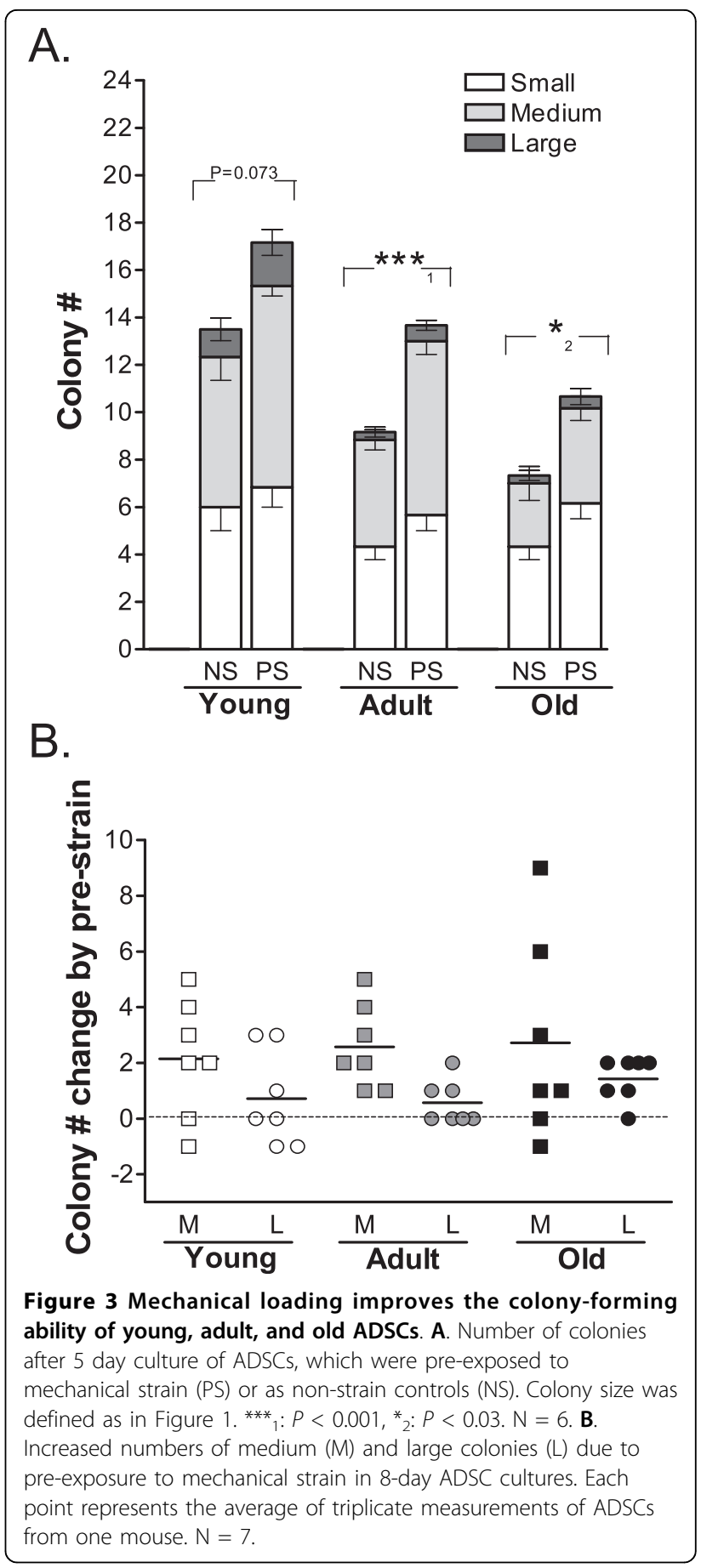

increase in un-induced cells, indicating the validity of our analysis. This experiment also showed that prestrain treatment failed to result in significant increases in ARS staining. Detailed pictures of ARS colonies and ethidium bromide staining are shown in Figure 5B. The expression of osteogenic marker genes in adult ADSCs, after normalization to GAPDH expression, was also not enhanced by pre-strain (Figure 5C). Therefore, we 

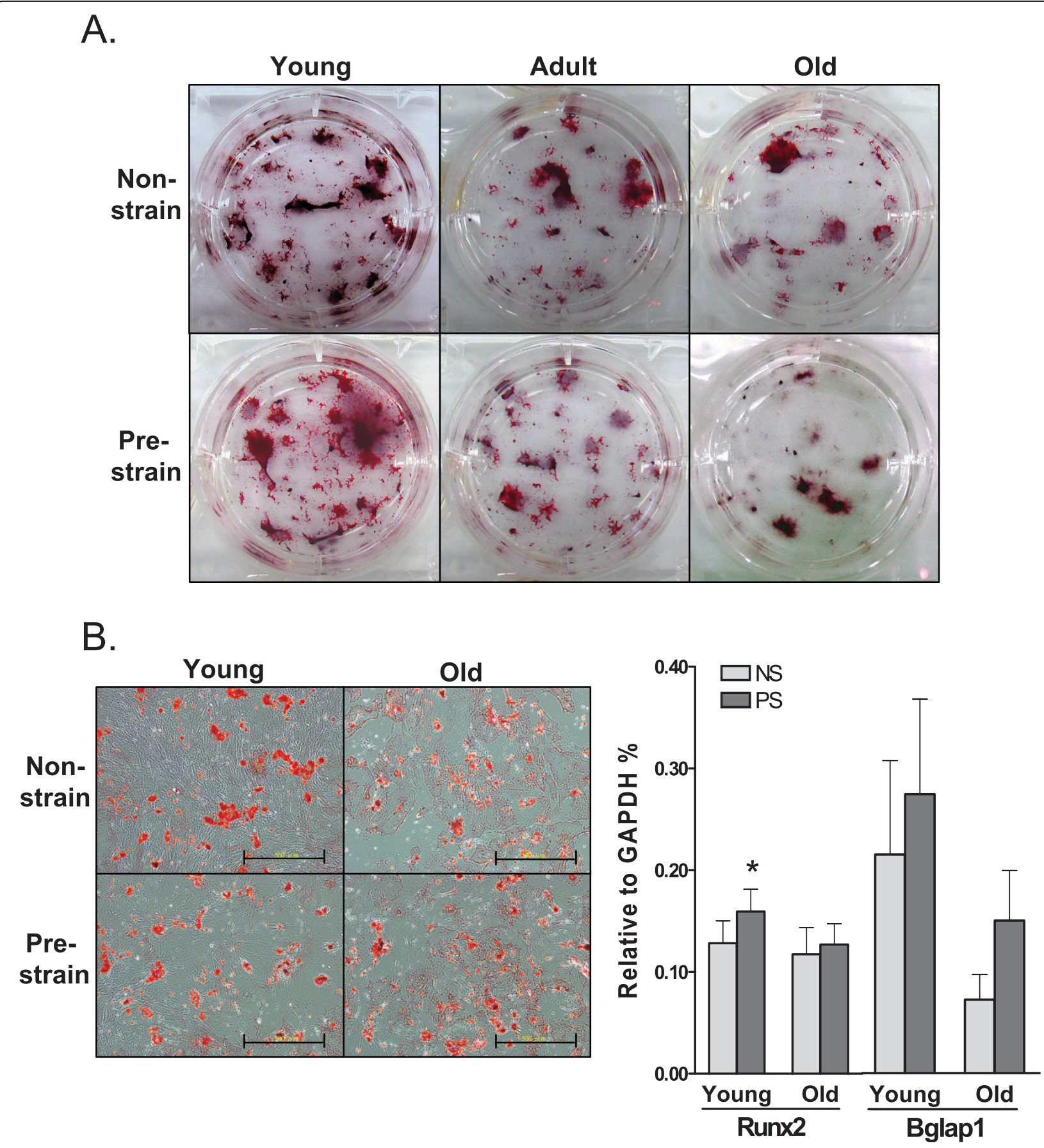

Figure 4 A. ARS staining for calcium deposition in osteogenically-induced young, adult, and old ADSCs with or without pre-exposure to mechanical strain. B. Left Osteogenic colonies from young and old ADSCs with or without pre-strain. Young ADSCs have stronger ARS staining than old cells. No difference in ARS staining caused by pre-strain was found. Scale bars $=500 \mu \mathrm{m}$. Right The expression of Runx2 and Bglap1 in young and old ADSCs with or without pre-strain. $N=8 .{ }^{*}: P<0.05$, compared to non-strained young ADSCs. 


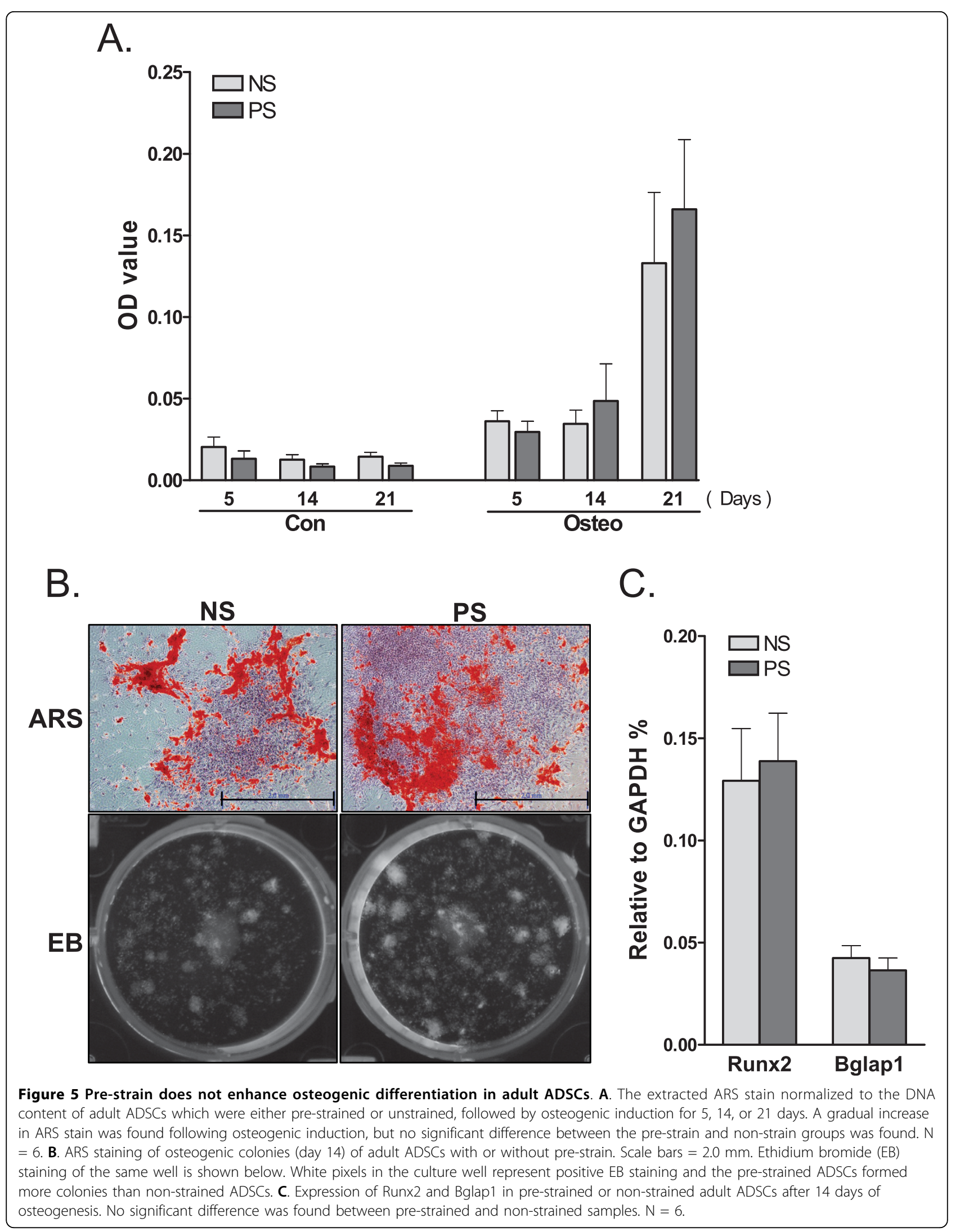


conclude that pre-strain increases the proliferation of ADSCs, and that the increase in ARS-stained colonies formed by pre-strained ADSCs is due to an increase in the number of colonies formed, but not an increase in the osteogenic potential of the colonies after mechanical loading.

\section{Pre-exposure to mechanical strain inhibits adipogenic differentiation in aging ADSCS}

In contrast to the result in osteogenesis, pre-strain significantly reduced adipogenic differentiation in ADSCs, as shown by the Oil-Red $\mathrm{O}$ staining result in Figure 6A. Very few oil droplets were found in young ADSCs after induction, compared to adult and old ADSCs. There were much fewer oil droplets in pre-strained adult and old ADSCs than in non-strained cells. The expression of AP2 and PPAR $\gamma$ in old ADSCs after adipogenic induction for 14 days was significantly reduced by pre-strain, indicating a strong inhibitory effect of mechanical loading on adipogenesis (Figure 6B). The effect of aging and pre-strain on ADSCs is summarized in Table 1.

\section{Stronger inhibition of adipogenesis by simultaneous mechanical loading and differentiation induction}

In the pre-strain experiments, ADSCs were passaged after $48 \mathrm{~h}$ of mechanical loading and cultured for an additional five days in maintenance medium without mechanical stimulation, followed by differentiation induction. To explore the effect of mechanical loading during differentiation induction, we applied the same pattern of mechanical stretching to ADSCs simultaneously with adipogenic induction. The Oil-Red O staining shown in Figure 7A demonstrates a strong inhibition of adipogenesis in both adult and old ADSCs by a simultaneously applied mechanical loading. A significant reduction in marker expression by strain was observed in adult and old ADSCs under adipogenic induction for only five days (Figure 7B). The differing abilities of the two mechanical loading methods in inhibiting AP2 and PPAR $\gamma$ expression are shown in Figure 8, where simultaneously applied mechanical strain during adipogenic induction significantly reduced AP2 expression in young, adult, and old ADSCs, while pre-exposure to mechanical strain only reduced AP2 and PPAR $\gamma$ expression in old ADSCs. In addition, we also examined the effects of simultaneous mechanical stretching and osteogenic induction in adult ADSCs, and obtained results similar to those of the pre-strain study (data not shown).

To explore the effect of stretching magnitude on the ADSC responses to loading, we applied 0.5\% (low), 2\% (medium), and 10\% (high) stretching during adipogenic induction in adult ADSCs and compared the results of Oil-Red staining and expression of adipogenic marker genes. As shown in Figure 9A, ADSCs that were subjected to $10 \%$ or $2 \%$ stretching presented fewer oil droplets than non-strained cells and the cells with $0.5 \%$ stretching. ADSCs subjected to medium or high strain also presented reduced AP2 and PPAR $\gamma$ expression (Figure 9B). Therefore, our results suggest that ADSC adipogenesis was reduced only by medium and high strain, but not by low strain.

\section{Discussion}

The purpose of this study is to investigate the behavioral change of ADSCs in various degrees of aging with respect to self-renewal and differentiation induced by chemical cues. Another focus of this study is to explore improvement of the declined functions in aged ADSCs by mechanical loading. That ADSCs responded to $10 \%$ strain was previously reported $[23,24]$, therefore, we used $10 \%$ strain in most experiments as the starting loading magnitude. We demonstrated that the result of mechanical loading in ADSCs depends on magnitude of the applied strain in which high strain (2-10\%) significantly reduces adipogenesis and low strain (0.5\%) has no effect (Figure 9). It should be noted that ADSCs in the mouse gonadal fat pad are not likely to experience $10 \%$ stretching in vivo, however, this magnitude of stretching is within the physiological range of loading for MSCs in muscular and peri-vascular tissues. We conducted our current study in ADSCs to take advantage of the ease of isolating large quantities of these cells from adult and aged animals. Further studies will be in need to verify that the observed results can be extrapolated to MSCs from other tissues as well as to the study of other forms of mechanical loading, using strain of various magnitudes, frequency, loading patterns, and durations.

Most current studies on MSCs have used established clones in which MSCs were selected through at least several rounds of passage and expansion. Aging (senescence) of MSCs in in vitro culture and loss of differentiation potential after the sixth passage has been demonstrated previously [25]. In this study, we chose to study primary ADSCs culture in very early passages (P0P3) in order to explore functional differences in ADSCs of various ages in the absence of artifacts likely contributed by clonal selection and expansion in long-term cultures. SVF from adipose tissue is known to contain primary cells with high degree of heterogeneity. We found that more than $50 \%$ of P0 cells after in vitro culture for one week exhibited the CD34 /CD45 /CD105 ${ }^{+} / \mathrm{CD} 3^{+} / \mathrm{CD}{ }^{+}$surface markers, which are the most accepted surface markers for MSCs (data not shown). It should also be noted that $>95 \%$ of P6 young ADSCs were $\mathrm{CD} 105^{+}, \mathrm{CD}^{+} 3^{+}$, and $\mathrm{CD}^{+} 0^{+}$(data not shown). We failed to obtain ADSC colonies beyond P10 from 21month-old mice (data not shown), indicating that passage-related senescence increases rapidly in old ADSC culture. 


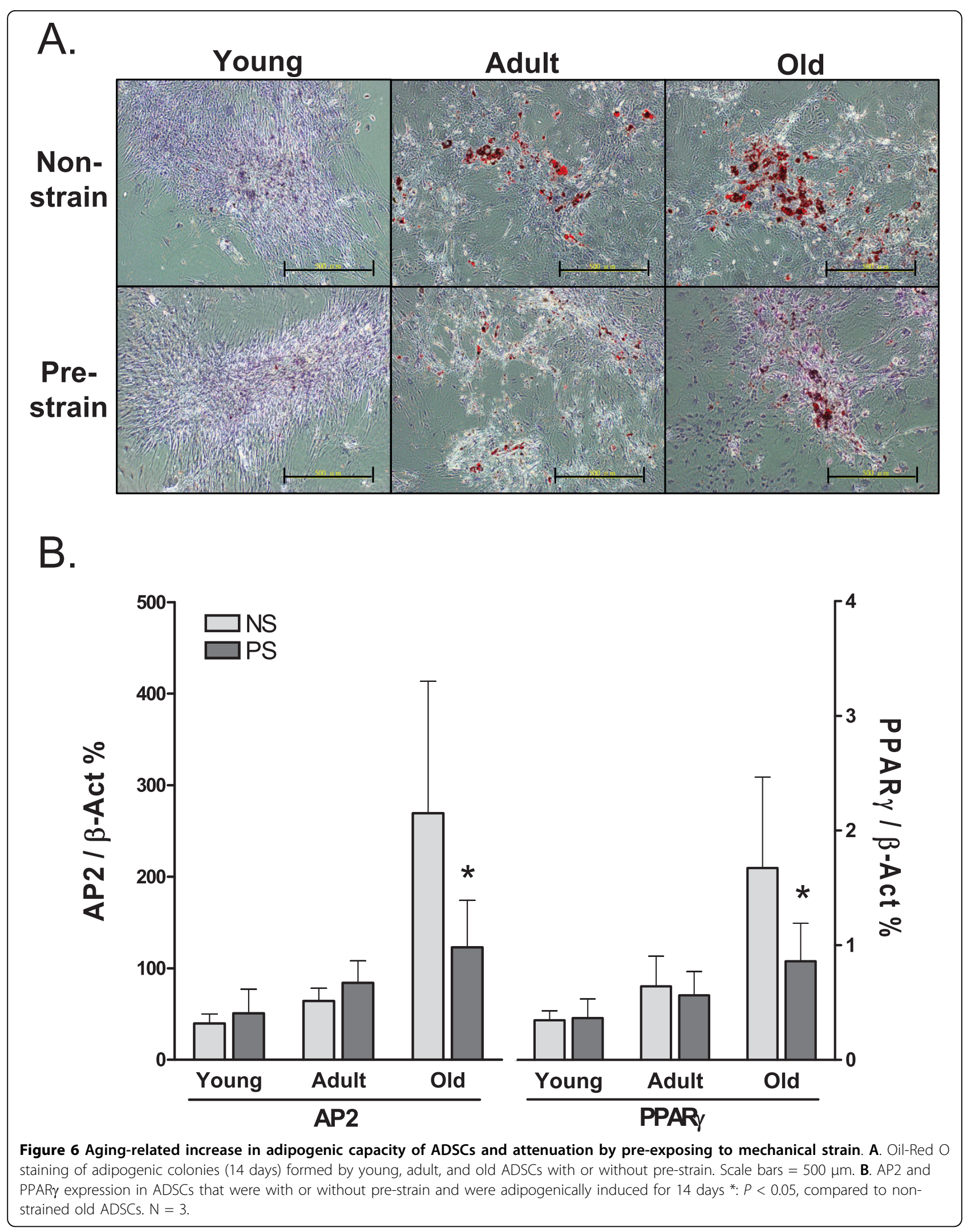


Table 1 Aging related changes in ADSCs and response to mechanical loading.

\begin{tabular}{lcc}
\hline & Aging & Mechanical Loading \\
\hline Self-renewal & $\downarrow$ & $\uparrow$ \\
Osteogenesis & - & - \\
Adipogenesis & $\uparrow$ & $\downarrow$ \\
\hline
\end{tabular}

$\uparrow$ denotes an increase, $\downarrow$ denotes a decrease, and - denotes no change. The change in myogenesis by aging was only partially studied in this study, thus, not reported here.

Short-term mechanical loading that simultaneously activates many mitotic signaling pathways is a strong inducer for cell proliferation [26]. But, long-term mechanical loading (more than $24 \mathrm{~h}$ ), which has not been as extensively studied as short-term loading, is less mitogenic [23,27]. We purposely investigated the cellular responses to long-term stretching $(48 \mathrm{~h})$ to distinguish the responses as the true adaptation of cells to mechanical loading from transient reactions to sudden changes in mechanical environment. Immediately following mechanical stretching, instead of an increase, a slight decrease (less than 5\%) in the number of ADSCs was found (data not shown). Thus, it is possible, also as a limitation for similar membrane stretching systems that mechanical stretching selects for cells that firmly adhere to the elastic membrane and allows the detached cells to die of anoikis. As a result, firmly adhered cells would be enriched following mechanical loading. Because the cell loss after strain was not significant when compared to the whole population, we believe that anoikis does not play an important role in our result. Yet, cell adhesion to substrate is an inherent cellular property that may be associated with self-renewal and differentiation potential. Indeed, BMMSCs of various sizes and different morphologies have been shown to exhibit different adhesion property to substrate [28]. In a separate report, these heterogeneous cells also display different selfrenewal abilities and express distinct surface markers [29]. In addition, the role of adhesion to substrate in dictating MSC differentiation potential was recently demonstrated [30]. Therefore, a change in ADSC adhesiveness to the substrate following mechanical loading should be investigated further. The finding that $48 \mathrm{~h}$ of pre-strain results in increased ADSC proliferation after replating (Figure 3) is intriguing to us. We did not find significant changes in the surface marker expression in ADSCs after applying strain (data not shown), therefore, the increase in proliferation following strain was not likely due to a selection for $\mathrm{CD} 105^{+} / \mathrm{CD} 3^{+} / \mathrm{CD} 90^{+}$cells during mechanical loading.

Because of a clear increase of calcium deposition in pre-strained ADSCs as well as in young cells, we originally hypothesized that aging would reduce, and mechanical stretching would enhance the osteogenesis capacity of ADSCs. An increase in calcium deposition can result from an increase in the number of total colonies without increasing the percentage of contained osteoblast colonies, or from an increase in both total colony number and the ratio of contained osteogenic colonies. While the former only results from an increase in proliferation, the latter represents a true increase in osteogenic capacity. By using adult ADSCs as a model, we demonstrated that in pre-strained cells, calcium deposition, when normalized to the DNA content, was not increased by strain as compared to non-strained cells. This result corroborates with the real time PCR data, which showed that bone marker gene expression did not increase as a result of pre-strain. The conclusion that the osteogenic potential of ADSC colonies was not increased by pre-strain is analogous to the result that no loss of osteogenic potential occurred in old ADSCs. Our finding in murine ADSCs is in agreement with previous reports that the osteogenic potential of human ADSCs from old donors was not reduced [13,31]. In the literature, conflicting reports on the relative osteogenic potential of BMMSCs and ADSCs exist, and more findings indicate that ADSCs have an inferior osteogenic potential relative to BMMSCs [32-34]. Thus, it is also possible that we failed to detect changes in osteogenic potential due to aging or mechanical loading in ADSCs, while mechanical loading induces osteogenesis in BMMSCs in other reports, may result from the intrinsically lower osteogenic capacity of ADSCs.

The adipogenic program is regulated by multiple signaling pathways and involves the activation of numerous transcription factors. PPAR $\gamma$ serves as the pivotal transcription factor in adipogenesis. Several previous reports demonstrating that mechanical stretching inhibits PPAR $\gamma$ signaling in 3T3-L1 cells [35], BMMSCs [14] and skeletal myoblasts [36] may provide insight into the mechanism that underlies inhibition of adipogenesis by mechanical loading. We also found that a change in AP2 expression, previously considered a late marker of adipocytes, precedes the change in PPAR $\gamma$ expression induced in ADSCs by both aging and mechanical loading (10\% strain). This result suggests that changes in PPAR $\gamma$ gene expression may not adequately reflect changes in PPAR $\gamma$ signaling, which can be better measured by the expression of its target genes. Indeed, AP2 is a PPAR $\gamma$ target gene $[37,38]$. We are currently examining the molecular basis of the inhibition of PPAR $\gamma$ signaling and reduction in adipogenesis as a result of mechanical strain. Two mechanical loading methods, pre-strain (PS) and simultaneous adipogenic induction and strain (AS), were compared in this study. It is not surprising to observe a stronger inhibitory effect of AS on adipogenesis, considering that the mechanical loading activates known signaling pathways leading to 


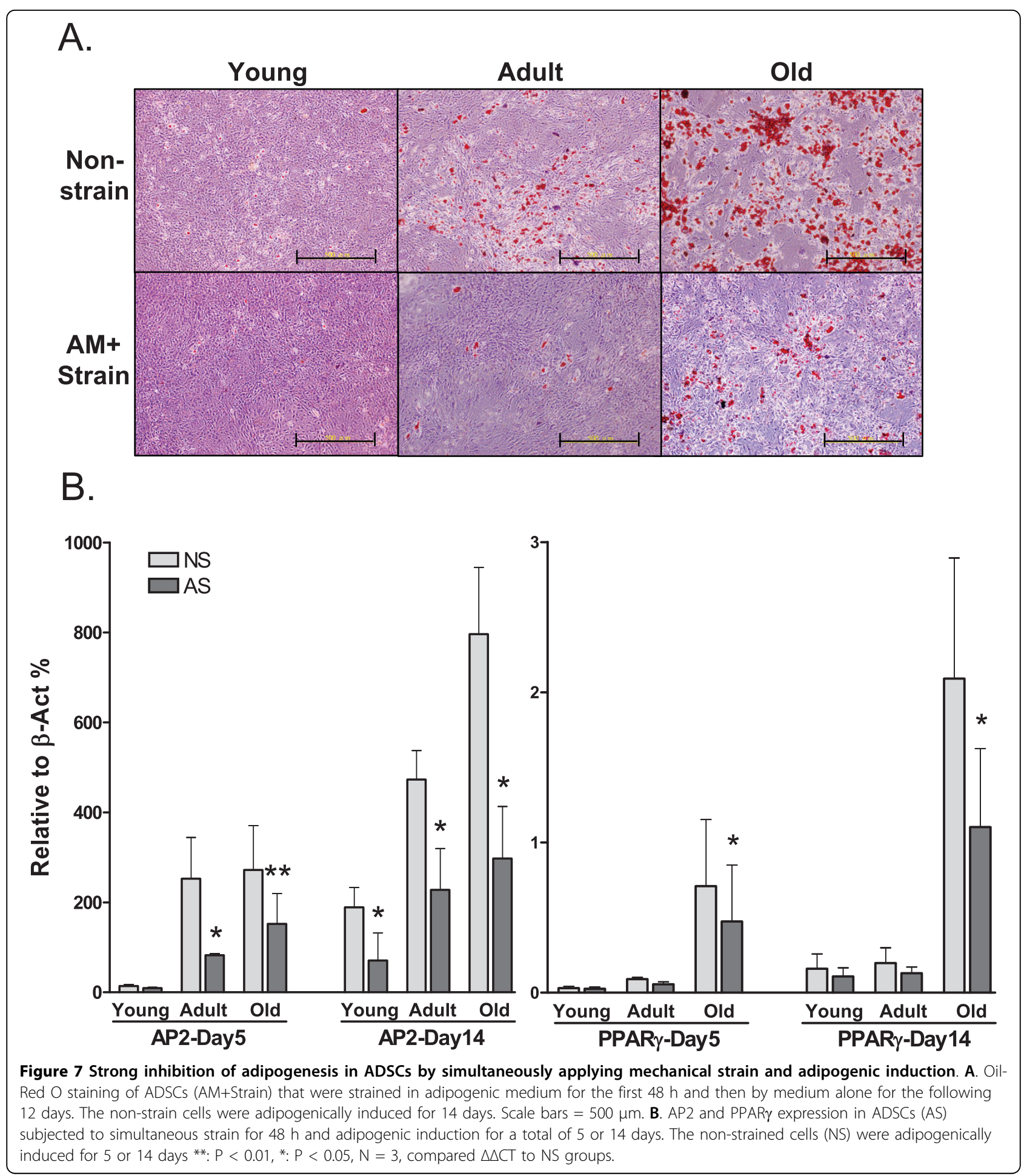

adipogenesis inhibition. We have found activation of non-canonical Wnt signaling by mechanical strain, involving calcium/calmodulin-dependent kinase II and nemo-like kinase, in various precursor cell lines and primary cells [39]. Therefore, inhibition of adipogenesis as a result of mechanical loading may be mediated by both canonical ( $\beta$-catenin dependent) [21] and non-canonical Wnt pathways.

\section{Conclusions}

Our results demonstrate an aging-dependent loss of selfrenewal and increased propensity for adipogenesis in old 


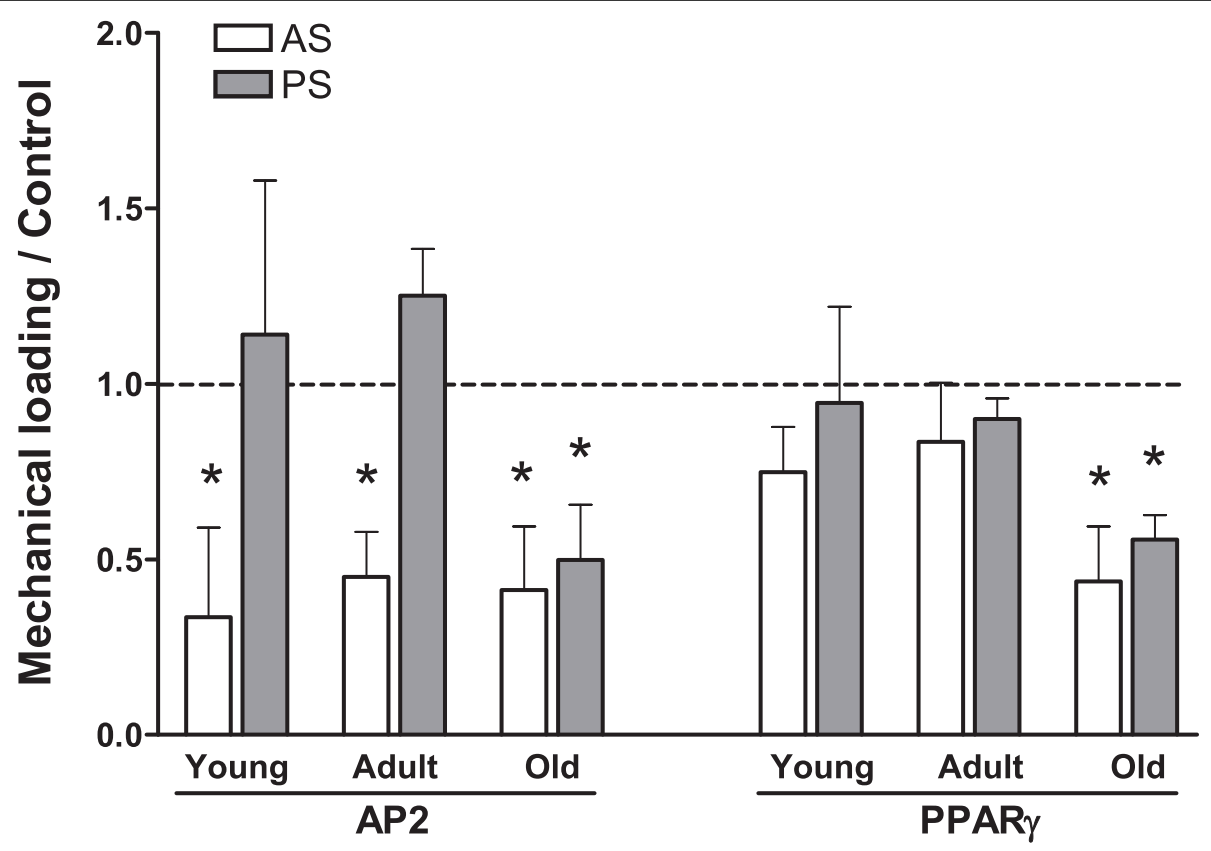

Figure 8 Comparison of the effect of pre-strain (PS) or simultaneous strain and adipogenic induction (AS). The levels of AP2 and PPAR $\gamma$ were normalized to $\beta$-actin and compared to the levels in NS groups that were induced for 14 days. Simultaneous strain applied during adipogenic induction was more effective at reducing adipogenic potential in ADSCs. *: $\mathrm{P}<0.05$, compared to $1 . \mathrm{N}=3$.

ADSCs and a positive effect of mechanical loading that counteracts the aging factor. These findings are important for the further study on stem cell mechanobiology and reveal the benefits and potential of combining an $e x$ vivo mechanical loading regime with autologous ADSC transplantation in treatment for a variety of aging related diseases.

\section{Methods}

\section{Chemicals and culture medium}

All chemicals used in this study were purchased from Sigma-Aldrich unless otherwise specified. All culture medium and reagents were purchased from Gibco-Invitrogen unless otherwise specified.

\section{Animals and ADSC isolation}

All animal experiments were conducted in accordance with accepted standards of animal care and were approved by the Institutional Animal Care and Use Committee of the National Health Research Institutes in Taiwan. Male FVB/NarL mice were used in this study and were grouped into young (8-10 weeks), adult (5 months), and old (21 months) groups. After euthanization by $\mathrm{CO}_{2}$, gonadal fat pads were isolated and digested by following a published protocol with minor modifications [40]. In short, fat pieces were digested with $0.2 \%$ collagenase for $30 \mathrm{~min}$ at $37^{\circ} \mathrm{C}$, followed by two, $5 \mathrm{~min}$ centrifugations at $260 \times \mathrm{g}$ to remove the adipocytes in supernatant. Following RBC lysis with $0.83 \% \mathrm{NH}_{4} \mathrm{Cl}$, the cells were washed twice with PBS, resuspended in maintenance medium, and plated in culture dishes for $4 \mathrm{~h}$ to remove non-adherent cells. The attached cells were considered SVF of P0 ADSCs. The isolated SVF was used immediately for experiments or cryopreserved for future studies. SVFs were cultured in maintenance medium consisting of $\alpha \mathrm{MEM}$ supplemented with $10 \%$ fetal bovine serum and $1 \%$ penicillin-streptomycin at $37^{\circ} \mathrm{C}$ in a humidified with $5 \% \mathrm{CO}_{2}$, and the medium was changed every $72 \mathrm{~h}$.

\section{Application of mechanical strain}

A Flexcell $4000 \mathrm{~T}$ tensile system (Flexcell INT) with a 25 $\mathrm{mm}$ post was used to generate equibiaxial strain. $2.5 \times$ $10^{5}$ P2 ADSCs were plated in each well of a type 1 collagen-coated, flexible bottom plate (Flexwell) at $2.8 \times$ $10^{4} \mathrm{cells} / \mathrm{cm}^{2}$ and cultured for another $16 \mathrm{~h}$, followed by a change to fresh medium before loading to the strain system. The mechanical stretching was applied as sinusoidal wave of $0.5 \mathrm{~Hz}$ for $48 \mathrm{~h}$ with a peak strain of $0.5 \%, 2 \%$, or $10 \%$ as indicated in the text. The medium in the plate of non-strain controls was also changed and placed in the same incubator as the strain samples. At the end of mechanical loading, ADSCs were collected by trypsin/EDTA, counted, and replated in 6-well plates for colony forming and differentiation assays. In studies with simultaneous mechanical strain and differentiation induction, cells were incubated with osteogenic or adipogenic medium at the beginning of the mechanical 


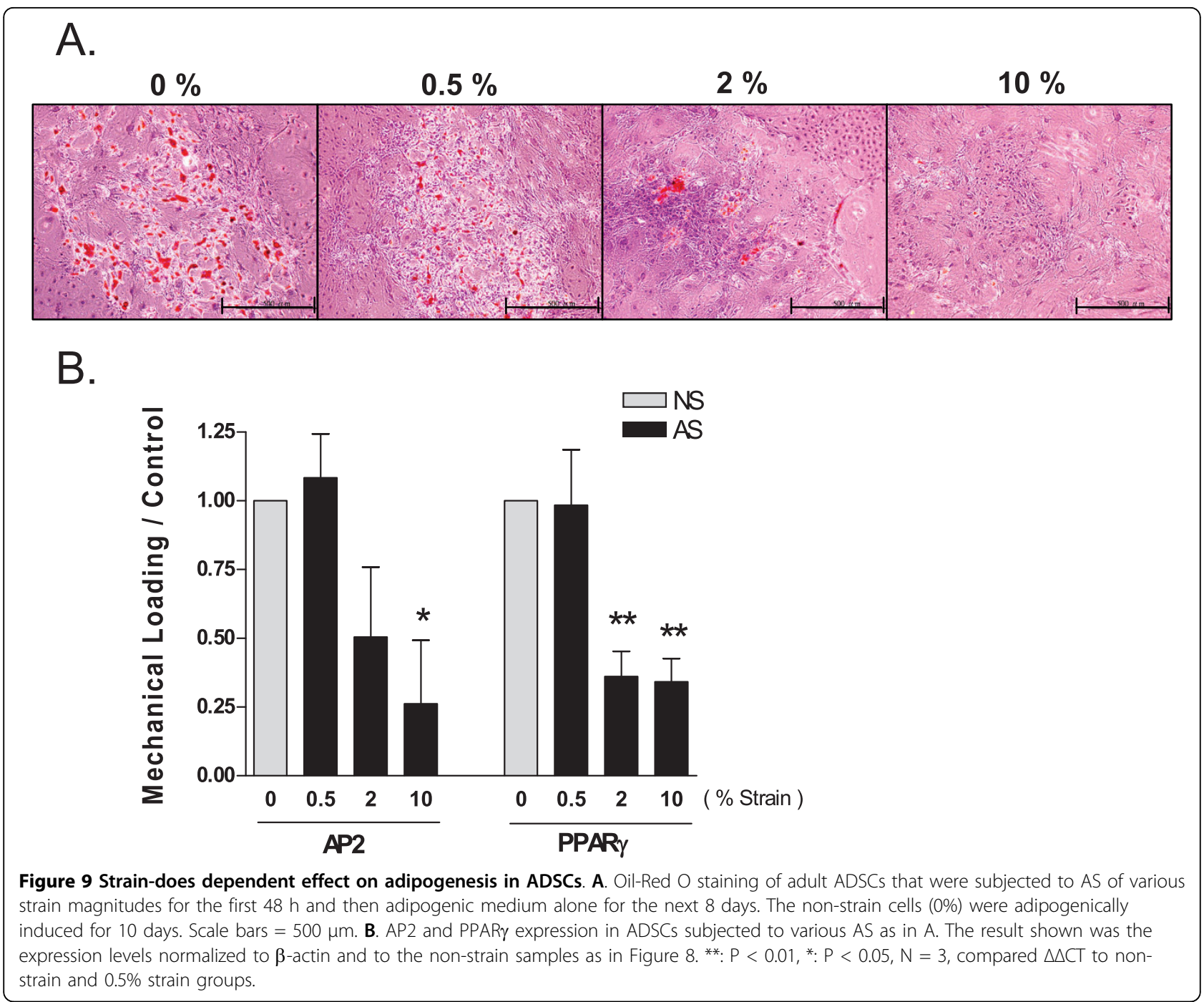

loading as described above, followed by a change to fresh differentiation medium at the end of loading and continued incubation as indicated in the text with medium changed every $72 \mathrm{~h}$.

\section{Doubling time measurement and colony-forming assay}

To measure the doubling time and colony-forming ability of ADSCs, 5000 cells were initially plated in each well of 6-well plates and cultured for various periods using maintenance medium. A cluster of cells consisting of at least five cells was considered a colony. The number of small (5-10 cells), medium (10-50 cells), and large ( $>50$ cells) colonies formed by ADSCs in each well was counted.

\section{Osteogenic induction and Alizarin Red S or von Kossa staining}

Osteogenic differentiation was induced using a previously described method [41]. In short, $2.5 \times 10^{4}$ ADSCs were seeded into each well of a 6-well plate, cultured for five days with maintenance medium, and then changed to differentiation induction medium. Osteogenic differentiation was induced by treating ADSCs with induction medium consisting of $100 \mathrm{nM}$ dexamethasone, $10 \mathrm{mM}$ sodium $\beta$-glycerophosphate, $0.05 \mathrm{mM} \mathrm{L}$-ascorbic acid-2-phosphate (Fluka), and 10\% fetal calf serum (FCS) in $\alpha$ MEM twice a week for 5,14 , and 21 days. For un-induced controls, cells were kept in maintenance medium. The degree of osteogenic differentiation was assessed by von Kossa or Alizarin Red S (ARS) staining for $\mathrm{Ca}^{2+}$ deposition using previously described protocols [42,43]. After washing with calcium and phosphate-free saline, $70 \%$ ethanol-fixed cells were stained for 3 min with a $2 \%$ ARS solution $(\mathrm{pH} 4.2)$ at room temperature followed by washing with water and incubation with PBS for 15 min. To quantify the ARS staining result, the deposition was extracted by $10 \%$ (w/ v) cetylpyridinium chloride in $10 \mathrm{mM}$ sodium phosphate ( $\mathrm{pH}$ 7.0) at room temperature for $1 \mathrm{~h}$, and the ARS 
concentration in the extraction buffer was determined by measuring the absorbance at $562 \mathrm{~nm}$. The absorbance values were normalized to the intensity of the ethidium bromide staining, which provides an estimation of the total DNA content of cells in a culture well. A standard curve correlating the intensity of ethidium bromide staining to the cell number in the well was established in parallel to correct for the factor used in normalization.

\section{Myogenic induction and Liu's stain}

Myogenic differentiation was induced using a previously described protocol [44]. In short, sub-confluent cells were induced by $10 \mu \mathrm{M} 5$-azacytidine for 21 days. Myogenic differentiation was examined with Liu's stain for the appearance of multinucleated myotubes.

\section{Adipogenic induction and Oil-Red $O$ stain}

Adipogenic differentiation was induced as previously described [45] using induction medium consisting of 10 $\mu \mathrm{M}$ dexamethasone, $0.25 \mu \mathrm{M}$ 3-isobutyl-1-methylxanthine, $4 \mu \mathrm{M}$ recombinant human insulin, $10 \mu \mathrm{M}$ troglitazone, and 10\% FCS. Adipogenic differentiation was assayed by the formation of neutral lipid vacuoles stainable with Oil-Red O.

\section{Quantitative PCR for the measurement of osteogenic and adipogenic marker gene expression}

Following osteogenic or adipogenic induction, ADSCs were lysed with Trizol to isolate RNA, followed by cDNA synthesis. Quantitative gene expression analysis was performed for mouse Runx2 and Bglap1 (Osteocalcin, $O C$ ) and normalized to GAPDH expression using Taqman gene expression assay (ABI system). The expression of adipogenic AP2 (Fabp4) and PPAR $\gamma$ was measured by Sybr Green-based real-time PCR, and the expression of $\beta$-actin was used as a control for constitutive expression. The sequences of probes and primers are listed in additional file 1.

\section{Statistical analyses}

All experiments were performed at least in triplicate. The results shown are the mean values with error bars representing the SEM. The two-tailed, unpaired Student's t-test was used for analysis unless otherwise specified. A $P$-value $<0.05$ was considered significant.

Additional file 1: Probes and primers used for quantitative PCR. The sequences of Taqman probes and PCR primers used for quantitative PCR are listed in this file.

Click here for file

[http://www.biomedcentral.com/content/supplementary/1471-2121-1118-S1.DOC]

\section{Acknowledgements}

This work was supported by Taiwan NHRI [ME097-PP02], [NM097-PP06], [ME097-SP07] to KML; Taiwan National Science Council [NSC-96-2627-B-400001] to KML; and ITRI [7356EA5100] to SCH.

\section{Author details}

${ }^{1}$ Biomedical Engineering Research Laboratories, Industrial Technology Research Institute, Hsinchu, Taiwan. ${ }^{2}$ Division of Medical Engineering Research, National Health Research Institutes, Zhunan Town, Miaoli, Taiwan. ${ }^{3}$ Department of Biomedical Imaging and Radiological Sciences, National Yang Ming University, Taiwan.

\section{Authors' contributions}

SCH conceived the idea, performed the study, and applied for the grant supporting this research. TCW contributed significantly to this work, performed the study, and prepared the figures. HCY and MRC maintained the animal colonies and performed the study. CML and WSC performed the study. KML conceived the idea, applied for the grant supporting this research, and wrote the paper. All authors read and approved the final manuscript.

Received: 13 July 2009

Accepted: 10 March 2010 Published: 10 March 2010

\section{References}

1. Drummond-Barbosa D: Stem cells, their niches and the systemic environment: an aging network. Genetics 2008, 180(4):1787-1797.

2. Di lorgi N, Rosol M, Mittelman SD, Gilsanz V: Reciprocal relation between marrow adiposity and the amount of bone in the axial and appendicular skeleton of young adults. J Clin Endocrinol Metab 2008, 93(6):2281-2286.

3. Meunier P, Courpron P, Edouard C, Bernard J, Bringuier J, Vignon G: Physiological senile involution and pathological rarefaction of bone. Quantitative and comparative histological data. Clin Endocrinol Metab 1973, 2(2):239-256.

4. Park KW, Halperin DS, Tontonoz P: Before they were fat: adipocyte progenitors. Cell Metab 2008, 8(6):454-457.

5. Khan WS, Adesida AB, Tew SR, Andrew JG, Hardingham TE: The epitope characterisation and the osteogenic differentiation potential of human fat pad-derived stem cells is maintained with ageing in later life. Injury 2009, 40(2):150-157.

6. Schaffler A, Buchler C: Concise review: adipose tissue-derived stromal cells-basic and clinical implications for novel cell-based therapies. Stem Cells 2007, 25(4):818-827.

7. Zhou S, Greenberger JS, Epperly MW, Goff JP, Adler C, Leboff MS, Glowacki J: Age-related intrinsic changes in human bone-marrowderived mesenchymal stem cells and their differentiation to osteoblasts. Aging Cell 2008, 7(3):335-343.

8. Bergman RJ, Gazit D, Kahn AJ, Gruber H, McDougall S, Hahn TJ: Age-related changes in osteogenic stem cells in mice. J Bone Miner Res 1996, 11(5):568-577.

9. D'Ippolito G, Schiller PC, Ricordi C, Roos BA, Howard GA: Age-related osteogenic potential of mesenchymal stromal stem cells from human vertebral bone marrow. J Bone Miner Res 1999, 14(7):1115-1122.

10. Zheng $H$, Martin JA, Duwayri Y, Falcon G, Buckwalter JA: Impact of aging on rat bone marrow-derived stem cell chondrogenesis. J Gerontol A Biol Sci Med Sci 2007, 62(2):136-148.

11. Roobrouck VD, Ulloa-Montoya F, Verfaillie CM: Self-renewal and differentiation capacity of young and aged stem cells. Exp Cell Res 2008, 314(9):1937-1944.

12. Moerman EJ, Teng K, Lipschitz DA, Lecka-Czernik B: Aging activates adipogenic and suppresses osteogenic programs in mesenchymal marrow stroma/stem cells: the role of PPAR-gamma2 transcription factor and TGF-beta/BMP signaling pathways. Aging Cell 2004, 3(6):379-389.

13. Shi YY, Nacamuli RP, Salim A, Longaker MT: The osteogenic potential of adipose-derived mesenchymal cells is maintained with aging. Plast Reconstr Surg 2005, 116(6):1686-1696.

14. David V, Martin A, Lafage-Proust MH, Malaval L, Peyroche S, Jones DB, Vico L, Guignandon A: Mechanical loading down-regulates peroxisome 
proliferator-activated receptor gamma in bone marrow stromal cells and favors osteoblastogenesis at the expense of adipogenesis. Endocrinology 2007, 148(5):2553-2562.

15. Byrne EM, Farrell E, McMahon LA, Haugh MG, O'Brien FJ, Campbell VA, Prendergast PJ, O'Connell BC: Gene expression by marrow stromal cells in a porous collagen-glycosaminoglycan scaffold is affected by pore size and mechanical stimulation. J Mater Sci Mater Med 2008, 19(11):3455-3463.

16. Qi MC, Hu J, Zou SJ, Chen HQ, Zhou HX, Han LC: Mechanical strain induces osteogenic differentiation: Cbfa1 and Ets-1 expression in stretched rat mesenchymal stem cells. Int I Oral Maxillofac Surg 2008, 37(5):453-458.

17. Wiesmann A, Buhring HJ, Mentrup C, Wiesmann HP: Decreased CD90 expression in human mesenchymal stem cells by applying mechanical stimulation. Head Face Med 2006, 2:8.

18. Park JS, Chu JS, Cheng C, Chen F, Chen D, Li S: Differential effects of equiaxial and uniaxial strain on mesenchymal stem cells. Biotechnol Bioeng 2004, 88(3):359-368.

19. Kurpinski K, Park J, Thakar RG, Li S: Regulation of vascular smooth muscle cells and mesenchymal stem cells by mechanical strain. Mol Cell Biomech 2006, 3(1):21-34

20. Kurpinski K, Chu J, Hashi C, Li S: Anisotropic mechanosensing by mesenchymal stem cells. Proc Natl Acad Sci USA 2006, 103(44):16095-16100.

21. Sen $B$, Xie Z, Case N, Ma M, Rubin C, Rubin J: Mechanical strain inhibits adipogenesis in mesenchymal stem cells by stimulating a durable betacatenin signal. Endocrinology 2008, 149(12):6065-6075.

22. Rubin CT, Capilla E, Luu YK, Busa B, Crawford H, Nolan DJ, Mittal V, Rosen CJ, Pessin JE, Judex S: Adipogenesis is inhibited by brief, daily exposure to high-frequency, extremely low-magnitude mechanical signals. Proc Natl Acad Sci USA 2007, 104(45):17879-17884.

23. Lee WC, Maul TM, Vorp DA, Rubin JP, Marra KG: Effects of uniaxial cyclic strain on adipose-derived stem cell morphology, proliferation, and differentiation. Biomech Model Mechanobiol 2007, 6(4):265-273.

24. Wall ME, Rachlin A, Otey CA, Loboa EG: Human adipose-derived adult stem cells upregulate palladin during osteogenesis and in response to cyclic tensile strain. Am J Physiol Cell Physiol 2007, 293(5):C1532-1538.

25. Bonab MM, Alimoghaddam K, Talebian F, Ghaffari SH, Ghavamzadeh A, Nikbin B: Aging of mesenchymal stem cell in vitro. BMC Cell Biol 2006, 7:14.

26. Chien S: Mechanotransduction and endothelial cell homeostasis: the wisdom of the cell. Am J Physiol Heart Circ Physiol 2007, 292(3):H1209-1224.

27. Lin K, Hsu PP, Chen BP, Yuan S, Usami S, Shyy JY, Li YS, Chien S: Molecular mechanism of endothelial growth arrest by laminar shear stress. Proc Natl Acad Sci USA 2000, 97(17):9385-9389.

28. Docheva D, Padula D, Popov C, Mutschler W, Clausen-Schaumann H, Schieker M: Researching into the cellular shape, volume and elasticity of mesenchymal stem cells, osteoblasts and osteosarcoma cells by atomic force microscopy. J Cell Mol Med 2008, 12(2):537-552.

29. Haasters F, Prall WC, Anz D, Bourquin C, Pautke C, Endres S, Mutschler W, Docheva D, Schieker M: Morphological and immunocytochemical characteristics indicate the yield of early progenitors and represent a quality control for human mesenchymal stem cell culturing. I Anat 2009, 214(5):759-767.

30. Oh S, Brammer KS, Li YS, Teng D, Engler AJ, Chien S, Jin S: Stem cell fate dictated solely by altered nanotube dimension. Proc Natl Acad Sci USA 2009, 106(7):2130-2135.

31. Khan WS, Adesida AB, Tew SR, Andrew JG, Hardingham TE: The epitope characterisation and the osteogenic differentiation potential of human fat pad-derived stem cells is maintained with ageing in later life. Injury 2009, 40(2):150-157.

32. Hayashi $\mathrm{O}$, Katsube $\mathrm{Y}$, Hirose $\mathrm{M}$, Ohgushi $\mathrm{H}$, Ito $\mathrm{H}$ : Comparison of osteogenic ability of rat mesenchymal stem cells from bone marrow, periosteum, and adipose tissue. Calcif Tissue Int 2008, 82(3):238-247.

33. De Ugarte DA, Morizono K, Elbarbary A, Alfonso Z, Zuk PA, Zhu M, Dragoo $\mathrm{J}$, Ashijan P, Thomas B, Benhaim P, et al: Comparison of multilineage cells from human adipose tissue and bone marrow. Cells Tissues Organs 2003, 174(3):101-109.

34. Im Gl, Shin YW, Lee KB: Do adipose tissue-derived mesenchymal stem cells have the same osteogenic and chondrogenic potential as bone marrow-derived cells?. Osteoarthritis Cartilage 2005, 13(10):845-853.
35. Tanabe $Y$, Nakayama K: [Mechanical stretching inhibits adipocyte differentiation of 3T3-L1 cells: the molecular mechanism and pharmacological regulation]. Nippon Yakurigaku Zasshi 2004, 124(5):337-344.

36. Akimoto T, Ushida T, Miyaki S, Akaogi H, Tsuchiya K, Yan Z, Williams RS, Tateishi T: Mechanical stretch inhibits myoblast-to-adipocyte differentiation through Wnt signaling. Biochem Biophys Res Commun 2005, 329(1):381-385.

37. Rival Y, Stennevin A, Puech L, Rouquette A, Cathala C, Lestienne F, DupontPasselaigue $E$, Patoiseau JF, Wurch T, Junquero D: Human adipocyte fatty acid-binding protein (aP2) gene promoter-driven reporter assay discriminates nonlipogenic peroxisome proliferator-activated receptor gamma ligands. J Pharmacol Exp Ther 2004, 311(2):467-475.

38. Yoshida K, Ono M, Koishi R, Furukawa H: Characterization of the $5^{\prime}$ regulatory region of the mouse angiopoietin-like protein 4 . Vet Res Commun 2004, 28(4):299-305.

39. Yu HC, Wu TC, Chen MR, Liu SW, Chen JH, Lin K: Mechanical stretching induces osteoprotegerin in differentiating $\mathrm{C} 2 \mathrm{C} 12$ precursor cells through non-canonical Wnt pathways. J Bone Miner Res.

40. Yamamoto N, Akamatsu H, Hasegawa S, Yamada T, Nakata S, Ohkuma M, Miyachi $E$, Marunouchi T, Matsunaga $\mathrm{K}$ : Isolation of multipotent stem cells from mouse adipose tissue. J Dermatol Sci 2007, 48(1):43-52

41. Jaiswal N, Haynesworth SE, Caplan Al, Bruder SP: Osteogenic differentiation of purified, culture-expanded human mesenchymal stem cells in vitro. J Cell Biochem 1997, 64(2):295-312.

42. Stanford CM, Jacobson PA, Eanes ED, Lembke LA, Midura RJ: Rapidly forming apatitic mineral in an osteoblastic cell line (UMR 106-01 BSP). J Biol Chem 1995, 270(16):9420-9428.

43. Zhang W, Yang N, Shi XM: Regulation of mesenchymal stem cell osteogenic differentiation by glucocorticoid-induced leucine zipper (GILZ). J Biol Chem 2008, 283(8):4723-4729.

44. Roura S, Farre J, Soler-Botija C, Llach A, Hove-Madsen L, Cairo JJ, Godia F, Cinca J, Bayes-Genis A: Effect of aging on the pluripotential capacity of human CD105+ mesenchymal stem cells. Eur J Heart Fail 2006, 8(6):555-563.

45. Kim YK, Choi HY, Kim NH, Lee W, Seo DW, Kang DW, Lee HY, Han JW, Park SW, Kim SN: Reversine stimulates adipocyte differentiation and downregulates Akt and p70(s6k) signaling pathways in 3T3-L1 cells. Biochem Biophys Res Commun 2007, 358(2):553-558.

doi:10.1186/1471-2121-11-18

Cite this article as: Huang et al:: Mechanical strain modulates agerelated changes in the proliferation and differentiation of mouse adipose-derived stromal cells. BMC Cell Biology 2010 11:18.

\section{Submit your next manuscript to BioMed Central and take full advantage of:}

- Convenient online submission

- Thorough peer review

- No space constraints or color figure charges

- Immediate publication on acceptance

- Inclusion in PubMed, CAS, Scopus and Google Scholar

- Research which is freely available for redistribution

Submit your manuscript at www.biomedcentral.com/submit
C Biomed Central 